Original Article

\title{
Surgical Outcome of Transpedicular Fixation for Degenerative Spondylolisthesis in Terms of Relief from Backache
}

\author{
Naeem-ul-Haq, Muhammad Ishaq, Musawer Khan, Adnan Ahmed, Gohar Ali \\ Department Neurosurgery, Mardan Medical Complex, Mardan - Pakistan
}

\begin{abstract}
Objective: To document the outcome of transpedicular fixation for degenerative spondylolisthesis in terms of relief from backache.
\end{abstract}

Materials \& Methods: This retrospective observational study was conducted in Mardan Medical Complex, Mardan. Static and dynamic spinal X-rays were used to diagnose degenerative spondylolisthesis. This research looked at how patients' pain levels, neurological state, and union improved after surgery. Patients with grade 5 spondylolisthesis, having congenital spinal anomaly, or who had previous spinal surgery were excluded from the study.

Results: In total 35 patients were included, $54.3 \%$ were male and $45.7 \%$ female. The primary reason for surgery was severe backache with 24 individuals having normal neurological status and 11 patients having some neurological abnormality. L5-S1 was the most commonly involved level in 24 patients. Meyerding grades were used to determine the slip grade. The average duration of follow-up was two years and five months, with a maximum of five years and a minimum of six months. 83 percent of patients had pain reduction, whereas neurological improvement was achieved in 64 percent of instances and surgical union was achieved in 91 percent of cases. There was no neurological impairment in the patients treated with transpedicular fixation. Significant differences exist between preoperative and postoperative GRS scores in the following pain categories: no pain, mild, server and excruciating.

Conclusion: Transpedicular fixation is a protected, simple, and successful procedure for patients with spondylolisthesis for pain relief in backache. It also contributes to surgical union and improvement in the neurological status of the patients.

Keywords: Spondylolisthesis, Meyerding grading, Transpedicular fixation.

Corresponding Author: Musawer Khan

Department of Neurosurgery, Mardan Medical Complex MTI, Mardan - Pakistan

Email: modestgaze@yahoo.com

Date of Submission: 15-07-2021

Date of Revision: 05-08-2021

Date of Acceptance: 31-08-2021

Date of Online Publishing: 30-09-2021

Date of Print: 30-09-2021
DOI: $10.36552 /$ pjns.v25i3.592

\section{INTRODUCTION}

Spondylolisthesis is a condition in which one vertebra slips relative to the other one as a result of structural and degenerative changes. Its causes are multifaceted: including disc degeneration, the anatomical orientation of the facet joints, iliolumbar arrangement, and ligament hyperlaxity. 
The most frequently performed surgical procedure is decompression, which may need a tailored surgical plan. ${ }^{1}$ Only decompression, on the other hand, can advance the slippage, which may result in pain or recurrence of neurological problems. As a result, lumbar fusion and fixation are regarded appropriate for spine stabilization and prevention of delayed degeneration. ${ }^{2}$ Spondylolisthesis was classified as dysplastic, ischemic, degenerative, traumatic, pathologic, and iatrogenic by Wiltse and Rothman. Meyerding grades I, II, III, IV, and V (spondylosis) are used to assess the degree of slide. Meyerding grades I and II are regarded as low, whereas Meyerding grades III, IV, and V are regarded as high. ${ }^{3}$ Grade I patients had a slipped vertebral body ranging from 0 to 25 percent of its diameter. Grade II represent a spondylolisthesis ranging between 25 to $50 \%$ of a vertebral body. Between 50 and $75 \%$ are grade III and grade IV is when the vertebral translation exceeds $75 \%$ but not beyond $100 \%$. Beyond $100 \%$ of vertebral slip is known as spondyloptosis. ${ }^{3}$

Lower back pain lasting three months affects around $29 \%$ of individuals. Spondylolisthesis is the forward or anterior displacement of one vertebra about the adjacent lower vertebra, which can result in low back pain. Congenital, isthmic, degenerative, traumatic, pathologic, and postoperative spondylolisthesis are all types of spondylolisthesis. ${ }^{4}$ Although spondylolisthesis can appear without symptoms, those with isthmic spondylolisthesis frequently suffer low back pain, neurologic symptoms, and/or radicular symptoms. The disease primarily affects the vertebrae in L3-S1 area. It most frequently affects the lumbosacral junction (L5-S1). Isthmic type ${ }^{2}$ is more prevalent in younger individuals and has an unknown etiology; the cause appears to be multifactorial, whereas degenerative type ${ }^{2}$ is more prevalent in the older age range. Spondylolisthesis occurs in 5-6\% of white males and $2-3 \%$ of females. In 82 percent of patients, the most prevalent site is $\mathrm{L} 5-\mathrm{S} 1$, followed by $\mathrm{L} 4-5$ in 11\%, L3-4 in 0.5 percent, and $\mathrm{L} 2-3$ in 0.5 percent. Spondylolysis is a weakening or stress fracture in the bone bridge connecting the upper and lower facet joints. It is the most severe grade of spondylolisthesis with complete slippage between upper and lower vertebrae. It is typically manifested by a backache that is increased by activity $^{5}$. A high pelvic incidence leads to increased shear stresses at the lumbosacral joint, which contributes to slippage. It has been established unequivocally that an aberrant sacropelvic orientation can disrupt the spine's sagittal equilibrium in L5-S1 spondylolisthesis. Using axially loaded magnetic resonance imaging, standing-related features such as impairment and physical functioning may be investigated (MRI). The intervertebral disc's angular instability also plays a larger role in the genesis and impairment of neurological compression. ${ }^{6}$

Patients with no neurologic impairment, acceptable or short-term pain, improvement with an exercise program or brace therapy, and a high level of patient comorbidity are candidates for conservative treatment. For low-grade and immobile slips, physiotherapy is preferred. However, regardless of spondylolisthesis grade, disc height, or mobility, surgical therapy is recommended, if this fails. The defect can be corrected through osteosynthesis or spondylodesis with a Neighboring Segment ${ }^{7-8}$ if operative therapy is considered. Bucks fusion, Morscher screw, and hook fixation, sublaminar wire (Scott's Operation), and bone grafting can all be used to achieve osteosynthesis with mobility segment preservation without the use of instruments. On grade I and II slips, laminoplasty is a treatment that can be performed. Posterolateral lumbar fusion (PLF), posterior lumbar interbody fusion (PLIF), transforaminal lumbar interbody fusion (TLIF), or combined anterior and posterior fusion ${ }^{7-8}$ are all options for spondylodesis or fusion with an adjacent segment. Fusion can be done with or without reducing the amount of material used. 
Spondylolisthesis surgery with transpedicle fixation is successful in the long run. ${ }^{8}$ With the introduction of transpedicular screw fixation methods and instrumentation systems, pedicle instruments implanted in tiny segments (a normal vertebra is fastened below and above a damaged lesion) have been brought into standard medical practice. The use of pedicle fixation, which can limit the number of segments of the spine, thereby reducing muscle and tendon damage and boosting the rate of synostosis, is one of the major purposes of pedicle screws, as previously established in studies. ${ }^{9}$

\section{MATERIALS and METHODS}

\section{Study Design and Setting}

Between May 2016 and February 2020, this descriptive study was undertaken with the permission of the patients and approval of the institutional review board, with a follow-up period of six months to five years. The study was conducted at the Department of Neurosurgery in Mardan Medical Complex, Mardan.

\section{Inclusion Criteria}

Thirty-five patients who required surgery were included in the study. Male and female patients between 20 and 80 years of age with a single level of spondylolisthesis, were included in the study. Those with minor disc alterations, with preserved disc heights were also included. Patients with severe lower back pain or with severe signs of radiculopathy, refractory to conservative measures who had consented to surgery were also included.

\section{Exclusion Criteria}

Those who were deemed unfit, unwilling, or receiving conservative treatment were excluded. Patients under 20 years of age, with local tissue problems requiring prior attention, having spondylolisthesis without symptoms, or minor backache spanning less than 3 months were excluded from the study. Those who could not be followed up for 6 months were also excluded. Grade V spondylolisthesis was also excluded. Patients with a congenital spinal deformity or who had undergone spinal surgery were also excluded from the study.

\section{Clinical and Surgical Management}

A panel of neurosurgery departments in Mardan Medical Complex performed the surgeries. Each patient was assigned a Proforma, and records were maintained in a custom-built Microsoft Access database. A prospective review evaluation of all treated fractures in the lumbar spine by surgery was conducted to determine several clinical factors such as age, gender distribution, and the source and extent of the damage. Patients underwent transpedicular fixation in small segmentation. Charts, operating notes, radiography performed before and after the surgery, computed tomography scans, MRIs, and visit records for a period of a minimum of six months were evaluated. Patients were administered general anesthesia and operated in prone positions. All pedicle screws were inserted using anatomical landmarks and leveled and positioned using an image intensifier. The study examined the improvement in pain severity, neurological state, and union following surgery.

\section{Assessment of Pain}

The graphic rating scale (GRS) was used to measure patients' pain before and after surgery. Clinical examinations, static/dynamic X-rays, and thin slice computed tomography were used to determine union (thin slice 3-D CT).

\section{Data Analysis}

The data were analyzed for frequencies, and descriptive statistics through SPSS, Version $25 . Z$ score calculations were done to find the significant/insignificant differences between 
preoperative and postoperative GRS scores in the following pain categories. A $p$-value of less than 0.05 was considered significant.

\section{RESULTS}

\section{Age Incidence}

The mean age was 48 years.

Table 1: Presentations of patients according to age distribution.

\begin{tabular}{ccc} 
Age in Years & Decade & No. of Patients \\
$21-30$ & $3^{\text {rd }}$ & 2 \\
$31-40$ & $4^{\text {th }}$ & 13 \\
$41-50$ & $5^{\text {th }}$ & 11 \\
$51-60$ & $6^{\text {th }}$ & 5 \\
$61-70$ & $7^{\text {th }}$ & 3 \\
$71-80$ & $8^{\text {th }}$ & 1 \\
\hline
\end{tabular}

\section{Gender Distributions}

In total 35 patients, $54.3 \%$ were male and $45.7 \%$ female. The majority of our 35 patients, were in their fourth or fifth decade (Table 1). A slight male predominance of 19 (54.3\%) male and 16 (45.7\%) females was observed.

\begin{tabular}{|lcc|}
\hline Table 2: Gender Distribution. & \\
\hline Gender & Number & Percentage \\
Male & 19 & $54.3 \%$ \\
Female & 16 & $45.7 \%$ \\
\hline
\end{tabular}

\section{Grades and their Occurrences}

Grade 1 spondylolisthesis sees Figure 1. Disc prolapse causing severe radiculopathy is shown in Figure 2 with no spondylolisthesis. See Figure 3

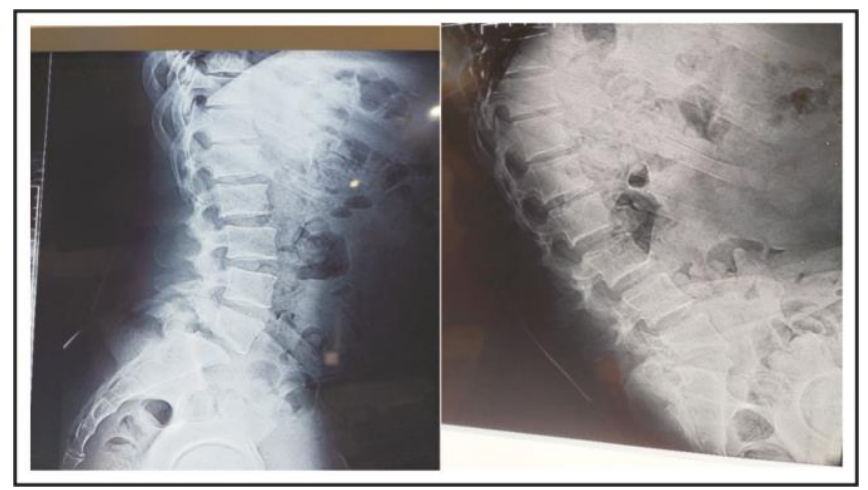

Figure 1: Pre-operative $x$-rays in dynamics of a 35 years old patient with L5 S1 spondylolisthesis due to pars fracture. for Pedicle screw fixation performed for root compression.

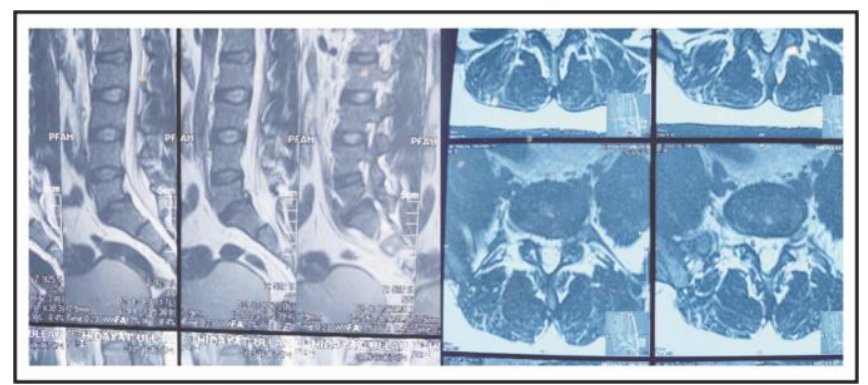

Figure 2: Pre-operative MRI of the same 35 years old patient with L5 S1 left-sided nerve root compression causing excruciating pain.

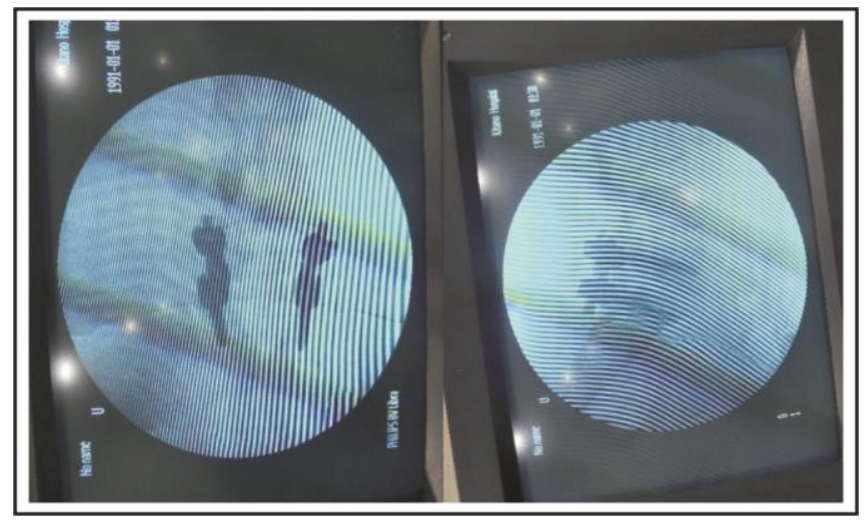

Figure 3: Intra-operative images of the same 35 years old patient which shows L5-S1 transpedicular screw fixation followed by left root decompression. Post-operatively the pain was resolved. 


\section{Clinical Presentation}

The pain was the primary reason for surgery inpatients. L5-S1 was affected in 24(68\%) instances, L4-L5 was affected in8 (23\%) cases, L3L4 was affected in 2 (6\%) cases, and L2-3 was affected in 1 (3\%) cases. Meyerding grades were used to determine the slip grade; 14 (40\%) patients had grade II, 11 (31\%) patients had grade I, 8 (23\%) patients had grade III, and 2 (6\%) patients had grade IV spondylolisthesis ${ }^{18}$ (Figure 4).

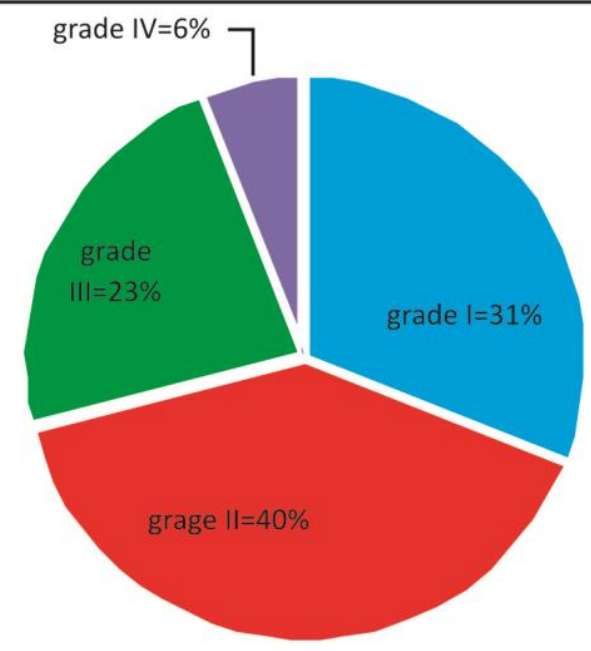

Figure 4: Percentage of patients presenting with spondylolisthesis pre-operatively.

\section{Grading of Pain}

Pedicle screw fixation (PSF) was employed in all cases. On the first postoperative day, patients were permitted to sit. They were gradually allowed to move by their neurological condition. The average duration of follow-up was two years and five months, with a maximum of five years and a minimum of six months. Pain relief was assessed on the graphic rating scale (GRS). 22 (63\%) patients exhibit no pain, $6(17 \%)$ patients complained of mild pain while 4 (11\%) and $3(9 \%)$ patients had moderate and severe pain respectively after surgery (Table-2). Significant differences exist between preoperative and postoperative GRS scores in the following pain categories: no pain ( $p$-value $<0.00001$ ), mild ( $p$ value 0.0106), server ( $p$-value $<0.00001$ ), and excruciating ( $p$ vale 0.02034 ).

Table 3: GRS grading of pain pre and postoperatively.

\begin{tabular}{|lcccc|} 
Pain (GRS) & Preoperative Postoperative & z- & p-value \\
No Pain & 0 & 22 & -5.66 & $<0.00001^{*}$ \\
Mild & 0 & 6 & -2.56 & $0.01046^{*}$ \\
Moderate & 4 & 4 & 0 & 1 \\
Severe & 26 & 3 & 5.58 & $<0.00001^{*}$ \\
Excruciating & 5 & 0 & 2.32 & $0.02034^{*}$ \\
\hline
\end{tabular}

*highly significant

\section{The Fate of Surgical Union}

We successfully negotiated surgical unions in 32 (91.4\%) patients postoperatively. Union was assessed on post-operative static/dynamic lumbosacral $x$-rays and 3D CT scans. There was no worsening in the neurological status of the patients after surgery. We had 1 patient with implant failure and 1 patient with wound infection post-operatively. ${ }^{15}$

\section{DISCUSSION}

Clinical prognosis in spondylolisthesis is highly dependent on firm fusion. In comparison to patients treated non-operatively, patients treated surgically for degenerative spondylolisthesis and concomitant spinal stenosis get significantly greater pain relief and functional improvement for a longer period. Fusion was accomplished in (91.4\%) patients. ${ }^{10}$ While there are several surgical techniques available, the decision to continue with decompression and fusion is determined on the patient's presenting symptoms and degree of instability. Symptomatic patients frequently appear with significant pain, neurological impairments, or deformity. In $83 \%$ of patients, we achieved significant pain alleviation. ${ }^{11}$

In spondylolysis, the lesion is a non-union of 
the pars interarticularis fatigue fracture. Because it retains the mobility segment and prevents anomalous pressures at surrounding levels, direct repair of the pars defect is preferable than fusion. Buck's fusion can be used to treat grade IV spondylolisthesis (Spondylolysis). In comparison to traditional in situ fusions, posterior pedicle screw-rod fixation had a greater rate of arthrodesis. At L5-S1, there is some reduction through pedicle screw and circumferential fusion, which can be accomplished totally via a posterior approach or via separate anterior and posterior methods. $^{12}$

A significant benefit of careful internal fixation over brief medicines is that early transpedicular instrumentation makes the patients mobile early, forestalling nerve harm by settling the spine. It shields harmed structures from external factors while expanding the chance of reclamation of neurological issues and supplanting the harmed constructions with fitting internal fixation apparatuses. Frankel et al announced that medicines utilizing stances would be sufficient and that it is feasible to make patients mobile in the wake of reestablishing their stability by longterm restoration. ${ }^{13} \mathrm{~A}$ dynamic stabilization, utilizing interspinous and pedicle screw-based devices, is another approach to treating lowgrade slippage.

Fusion of a high-grade spondylolisthesis can be done with or without spondylolisthesis reduction; however, reduction puts the exiting nerve root in danger. To treat high-grade slips without decreasing the spondylolisthesis, two cancellous bone screws can be inserted bilaterally through the pedicles of the lower vertebra into the body of the upper slipped vertebra. ${ }^{14}$ The surgical procedure Individuals with high-grade spondylolisthesis face a difficult and controversial surgical treatment. Even though transpedicular fixation produces a high fusion rate with some decrease, implant failure, and lower fusion rates have been reported. The surgical procedure Individuals with high-grade cancer are treated surgically. In the modern era, the treatment of high-grade spondylolisthesis usually consists of some spondylolisthesis reduction combined with pedicle screw instrumentation and circumferential fusion at L5-S1, which can be done entirely through a posterior approach or separately through anterior and posterior approaches. Additionally, the fibular strut grafts can be put anteriorly as part of an anterior/posterior treatment or posteriorly as part of a posterioronly procedure. ${ }^{15}$

\section{CONCLUSION}

Transpedicular instrumentation is a protected, simple, and successful procedure for patients with spondylolisthesis to relieve backache. Patients showed synostosis and fruitful recuperation from pain along with neurological improvements with no significant rate of complications. Yet, this study is restricted by its short subsequent followup period.

\section{Recommendations}

Transpedicular screw fixation provides effective fusion for the delineated spine and must be considered for relief of painful symptoms when conservative methods have failed. It should be done by an experienced surgeon for best outcome.

\section{REFERENCES}

1. Wiltse LL, Newman PH, Macnab I. Classification of spondylolysis and spondylolisthesis. Clin Orthop Relat Res. 1976; (117): 23-9.

2. Meyerding HW. Spondylolisthesis. Surg Gynecol Obstet. 1932; 54: 371-80.

3. Bridwell $\mathrm{KH}$. Surgical treatment of high-grade spondylolisthesis. Neurosurg Clin N Am. 2006; 17 (3): 331-8.

4. Jackson RP, Phipps T, Hales C, Surber J. Pelvic lordosis and alignment in spondylolisthesis. Spine (Phila Pa 1976), 2003; 28 (2): 151-60. 
5. Syrmou E, Tsitsopoulos PP, Marinopoulos D, Tsonidis C, Anagnostopoulos I, Tsitsopoulos PD. Spondylolysis: a review and reappraisal. Hippokratia. 2010; 14 (1): 17-21.

6. Labelle H, Mac-Thiong JM, Roussouly P. Spinopelvic sagittal balance of spondylolisthesis: a review and classification. Eur Spine J. 2011; 5: 6416.

7. Huang $K Y$, Lin RM, Lee $Y L$, Li JD. Factors affecting disability and physical function in degenerative lumbar spondylolisthesis of L4-5: evaluation with axially loaded MRI. Eur Spine J. 2009; 18 (12): 1851-7.

9. Pearson AM, Lurie JD, Blood EA, Frymoyer JW, Braeutigam $\mathrm{H}, \mathrm{An} \mathrm{H}$, et al. Spine patient outcomes research trial: radiographic predictors of clinical outcomes after operative or nonoperative treatment of degenerative spondylolisthesis. Spine (Phila Pa 1976). 2008; 33 (25): 2759-66.

10. Rajasekaran S, Subbiah M, Shetty AP. Direct repair of lumbar spondylolysis by Buck's technique. Indian J Orthop. 2011; 45 (2): 136-40.
11. Lee DY, Lee SH, Maeng DH. Two-level anterior lumbar interbody fusion with percutaneous pedicle screw fixation: a minimum 3-year follow-up study. Neurol Med Chir (Tokyo). 2010; 50 (8): 645-50.

12. Zagra A, Giudici F, Minoia L, Corriero AS, Zagra L. Longterm results of pediculo-body fixation and posterolateral fusion for lumbar spondylolisthesis. Eur Spine J. 2009; 18: 151-5.

13. Aunoble $S$, Hoste $D$, Donkersloot $P$, Liquois $F$, Basso Y, Le Huec JC. Video-assisted ALIF with cage and anterior plate fixation for L5-S1 spondylolisthesis. J Spinal Disord Tech. 2006; 19: 471-6.

14. $\mathrm{Yu} \mathrm{CH}$, Wang $\mathrm{CT}$, Chen $\mathrm{PQ}$. Instrumented posterior lumbar interbody fusion in adult spondylolisthesis. Clin Orthop Relat Res. 2008; 466: 3034-43.

15. Heft MV, Parker SR. An experimental basis for revising the graphic rating scale for pain. Pain. 1984; 19 (2): 153-61.

16. Marino R, Barros T, Biering-Sorensen $F$, Burns SP, Donovan $\mathrm{WH}$, Graves $\mathrm{DE}$, et al. International standards for neurological classification of spinal cord injury. J Spinal Cord Med. 2003; 26: S50-S56.

\section{Additional Information}

Disclosures: Authors report no conflict of interest.

Ethical Review Board Approval: The study was retrospective so ethical committee approval was not required.

Human Subjects: Consent was obtained by all patients/participants in this study.

\section{Conflicts of Interest:}

In compliance with the ICMJE uniform disclosure form, all authors declare the following:

Financial Relationships: All authors have declared that they have no financial relationships at present or within the previous three years with any organizations that might have an interest in the submitted work.

Other Relationships: All authors have declared that there are no other relationships or activities that could appear to have influenced the submitted work. 


\section{AUTHORS CONTRIBUTIONS}

\begin{tabular}{|l|l|l|}
\hline Sr.\# & Author's Full Name & Intellectual Contribution to Paper in Terms of: \\
\hline 1. & Naeem ul Haq & 1. Study design and methodology. \\
\hline 2. & Gohar Ali & 2. Paper writing, referencing, and data calculations \\
\hline 3. & Muhammad Ishaq & 3. $\quad$ Data collection and calculations \\
\hline 4. & Adnan Ahmed & 4. Analysis of data and interpretation of results etc. \\
\hline 5. & Muswar Khan & 5. Literature review and manuscript writing \\
\hline 6. & Naeem ul Haq & 6. Analysis of data and quality insurer \\
\hline
\end{tabular}

